\title{
Validação do The Alcohol, Smoking and Substance Involvement Screening Test (ASSIST) em Estudantes Universitários
}

\section{Validation of The Alcohol, Smoking and Substance Involvement Screening Test (ASSIST) Among University Students}

\author{
António Ramalho MOSTARDINHA $\triangle^{1}$, Ana BÁRTOLO², José BONIFÁCIO ${ }^{3,4}$, Anabela PEREIRA ${ }^{1}$ \\ Acta Med Port 2019 Apr;32(4):279-288 - https://doi.org/10.20344/amp.10650
}

\section{RESUMO}

Introdução: Este estudo objetiva adaptar culturalmente e explorar as propriedades psicométricas da versão portuguesa do the Alcohol, Smoking and Substance Involvement Screening Test em estudantes do ensino superior.

Material e Métodos: Estudo de validação, transversal, com dados recolhidos através de questionário composto por questões sociodemográficas e medidas para avaliação do consumo de substâncias (teste de Fagerström para dependência nicotínica; Drinking Motives Questionnaire - Revised e The Alcohol, Smoking and Substance Involvement Screening Test versão 3.1). A amostra foi constituída por 338 estudantes (51,8\% homens), com uma idade média de 20,6 anos (desvio-padrão = 3,4). A análise fatorial exploratória foi utilizada para o estudo da estrutura fatorial. Foram ainda avaliadas a consistência interna e a validade convergente.

Resultados: As subescalas 'Tabaco' e 'Cannabis' apresentaram-se ambas compostas por um único fator e a de 'Bebidas Alcoólicas' por dois fatores. A consistência interna variou entre 0,556 e 0,842 (a de Cronbach). Observaram-se associações estatisticamente significativas entre ser fumador, binge-drinking e motivos de consumos etílicos com consumos de risco (subescalas 'Tabaco', 'Bebidas Alcoólicas' e 'Cannabis').

Discussão: As associações observadas entre os consumos de risco, os motivos de consumos e os comportamentos de consumo, poderão ser explicadas pela relação entre o consumo de álcool (como parte da experiência académica) com o comportamento de consumo de substâncias e consumos concorrentes, fundamentando a adequada validade convergente observada. Ainda, a pressão de pares poderá influenciar estes comportamentos relativamente a consumos.

Conclusão: A versão em Português europeu do the Alcohol, Smoking and Substance Involvement Screening Test ('Tabaco', 'Bebidas Alcoólicas' e 'Cannabis') apresentou características psicométricas satisfatórias, mostrando ser uma ferramenta confiável para avaliar consumos de risco em estudantes universitários.

Palavras-chave: Estudantes; Fumar; Inquéritos e Questionários; Portugal; Transtornos Relacionados ao Uso de Álcool; Transtornos Relacionados ao Uso de Substâncias; Universidades

\section{ABSTRACT}

Introduction: The present study aims to culturally adapt and explore the psychometric properties of Portuguese version of the Alcohol, Smoking and Substance Involvement Screening Test, among university students.

Material and Methods: A validation, cross-sectional study, with data collected through a questionnaire comprised of sociodemographic and substance consumption measures (Fagerström test for Nicotine Dependence; Drinking Motives Questionnaire - Revised, and The Alcohol, Smoking and Substance Involvement Screening Test version 3.1). The sample was composed by 338 students (51.8\% male), with a mean age of 20.6 years old (standard deviation $=3.4$ ). To examine the factor structure, an exploratory factor analysis was performed. The internal consistency and convergent validity were also evaluated.

Results: The 'Tobacco' and 'Cannabis' subscales were composed by 1 factor and 'Alcoholic Beverages' by 2 factors. Internal consistency ranged between 0.556 and 0.842 (Cronbach's a). Statistically significant associations were observed between being a current smoker, binge-drinking and drinking motives with hazardous consumption (subscales 'Tobacco', 'Alcoholic Beverages' and 'Cannabis').

Discussion: The observed associations between hazardous consumption, consumption motives and behaviors, may be explained by the relation between alcohol consumption (as part of the academic experience) with substances consumption behavior and polydrug consumption, supporting the adequate convergent validity observed. Also, peer pressure may influence these consumption behaviors. Conclusion: The Portuguese version of the the Alcohol, Smoking and Substance Involvement Screening Test ('Tobacco', 'Alcoholic Beverages' and 'Cannabis') presented satisfactory psychometric characteristics, showing that it is an adequate instrument to assess hazardous consumption behaviors among university students.

Keywords: Alcohol-Related Disorders; Portugal; Smoking; Students; Substance-Related Disorders; Surveys and Questionnaires; Universities

\footnotetext{
1. Centro de Investigação em Didática e Tecnologia na Formação de Formadores. Departamento de Educação e Psicologia. Universidade de Aveiro. Aveiro. Portugal.

2. Centro de Investigação em Tecnologias e Serviços de Saúde. Departamento de Educação e Psicologia. Universidade de Aveiro. Aveiro. Portugal.

3. Unidade de Cuidados Saúde Personalizados Albergaria-a-Velha. Agrupamento de Centros de Saúde Baixo Vouga. Aveiro. Portugal.

4. Centro de Saúde Universitário. Serviços de Ação Social. Universidade de Aveiro. Aveiro. Portugal.

$\triangle$ Autor correspondente: António Ramalho Mostardinha. antonio.mostardinha@ua.pt

Recebido: 12 de abril de 2018 - Aceite: 22 de novembro de 2018 | Copyright @ Ordem dos Médicos 2019
} 
O consumo de substâncias, comum entre jovens adultos, nomeadamente os estudantes do ensino superior, ${ }^{1,2}$ revela-se uma área de atenção e atuação para os profissionais de saúde e educadores, pelos problemas associados aos consumos ${ }^{3,4}$ que poderão não ser percecionados pelos estudantes. ${ }^{5}$ Assim, os estudantes do ensino superior, devido à fase de transição em que se encontram, ${ }^{6}$ pelo desenvolvimento da identidade social $^{7}$ e pela pressão de pares, ${ }^{8}$ poderão estar mais suscetíveis a iniciar o consumo de substâncias (licitas e ilícitas).9,10

No contexto português, o Inquérito Nacional de Saúde de $2014^{11}$ apresentou uma prevalência de $20,0 \%$ de fumadores com 15 ou mais anos de idade. De igual modo, $34,5 \%$ de pessoas da mesma faixa etária afirmaram ter consumido diariamente bebidas alcoólicas no último ano. Ainda, com base nos dados constantes no Relatório Europeu sobre Drogas 2017, observou-se uma prevalência de $5,1 \%$ de consumo de cannabis em jovens adultos. ${ }^{12}$

\section{Problemática de estudo}

O consumo de substâncias entre os indivíduos atinge o seu pico entre os 18 e os 25 anos de idade tornando os estudantes universitários uma população alvo para realização de intervenções visando a promoção da saúde através do desenvolvimento e implementação de políticas saudáveis. ${ }^{13,14}$

O contexto ambiental em que estes estudantes se inserem, poderá influenciar a intenção e motivos de consumos de tabaco e álcool, ${ }^{15}$ associado, por vezes, ao consumo de cannabis (policonsumos). ${ }^{8,16}$ Ainda, devido aos motivos e intenções de consumo, pelo risco de consumos abusivos que esta população apresenta, torna-se necessário o estudo, desenvolvimento e validação de instrumentos que visem detetar/rastrear comportamentos de consumos abusivos ou de risco desta população.

Neste âmbito, o the Alcohol, Smoking and Substance Involvement Screening Test (ASSIST), desenvolvido pela Organização Mundial de Saúde (OMS), destaca-se de outros instrumentos como o the Alcohol Use Disorders Identification Test (AUDIT), ${ }^{17}$ pois objetiva detetar consumo abusivos de diferentes substâncias e problemas associados (tabaco, bebidas alcoólicas, cannabis, cocaína, estimulantes do tipo anfetaminas, inalantes, sedativos ou comprimidos para dormir, alucinogénios, opióides e outros). ${ }^{18}$

Consequentemente, torna-se pertinente a aplicação do ASSIST, pela necessidade de intervir nos estudantes do ensino superior, objetivando diminuir consumos de risco. Neste contexto, verifica-se na literatura que a aplicação de intervenções motivacionais breves, educação para a saúde, entre outros, têm sido utilizados na prevenção primária, reduzindo o risco do desenvolvimento de consequências negativas para a saúde. ${ }^{19,20}$ Por conseguinte, a utilização deste instrumento poderá ser vantajosa na medida em que outros estudos utilizaram o ASSIST nesta população, verificando a sua flexibilidade para a aplicação em diferentes contextos. ${ }^{21,22}$
Esta escala tem apresentado boas propriedades psicométricas em países como Austrália, Brasil, Reino Unido, África do Sul, México, Espanha França, entre outros. ${ }^{18,23-26}$ No entanto, que seja do nosso conhecimento, a validade da versão portuguesa do ASSIST ainda não foi explorada. Com base nessa lacuna, o presente estudo assumiu como objetivos específicos (i) adaptar culturalmente e (ii) explorar as propriedades psicométricas da versão portuguesa desta ferramenta usando uma amostra de estudantes universitários.

\section{MATERIAL E MÉTODOS}

\section{Participantes e procedimentos}

A recolha foi efetuada na Universidade de Aveiro, no ano letivo 2016/2017, sendo a população constituída por 8842 estudantes inscritos. O recrutamento dos participantes decorreu entre fevereiro e maio de 2017 e os dados recolhidos através de um questionário constituído por questões sociodemográficas e medidas de auto-relato para avaliação do consumo de substâncias. Estes questionários foram distribuídos por quatro estudantes do curso de Psicologia (com preparação prévia sobre a composição do questionário e orientações do seu preenchimento para esclarecer possíveis dúvidas dos inquiridos).

Da amostragem por conveniência, obtiveram-se 400 questionários, tendo-se excluído 62 por não terem respondido a escalas descritas na secção 'Instrumentos'. A amostra foi constituída por 338 estudantes, dos quais 51,8\% eram do sexo masculino, com uma média de idades de $20,6 \pm 3,4$ anos

Para a concretização do estudo foram obtidas as autorizações necessárias e todos os participantes incluídos assinaram um consentimento. A Tabela 1 apresenta a caracterização da amostra, na qual se descrevem as frequências absolutas e relativas (\%) de variáveis sociodemográficas e de variáveis relacionadas com consumos de substâncias.

Definiram-se como critérios de inclusão: (i) ter idades compreendidas entre os 18 e os 27 anos [primeira e segunda fase de transformação do jovem adulto]; ${ }^{27}$ (ii) frequentar o primeiro/segundo ciclos de estudos e (iii) ser falante nativo de língua portuguesa.

\section{Instrumentos \\ Descrição do ASSIST}

Relativamente à avaliação dos policonsumos e consequente aplicação de intervenção, o ASSIST encontra-se recomendado pela Norma n 36 de 2014 da Direcção-Geral da Saúde. ${ }^{28}$ Este é composto por 10 subescalas ('Tabaco', 'Bebidas Alcoólicas', 'Cannabis, 'Cocaína', 'Estimulantes do tipo Anfetaminas', 'Inalantes', 'Sedativos ou comprimidos para dormir', 'Alucinogénios', 'Opióides' e 'Outros'). A primeira questão é de triagem (consumir ou alguma vez ter consumido determinada substância). As questões 2 a 7 estão relacionadas com a avaliação do envolvimento com determinada substância (identificação do nível de risco que o seu comportamento de consumo apresenta). 
Tabela 1 - Medidas descritivas das características sociodemográficas e de consumo de substâncias na amostra $(n=338)$

\begin{tabular}{|c|c|c|}
\hline Variável & & n (\%) \\
\hline \multicolumn{3}{|l|}{ Caracterização sociodemográfica } \\
\hline \multirow[t]{2}{*}{ Sexo } & Masculino & $175(51,8)$ \\
\hline & Feminino & $163(48,2)$ \\
\hline \multirow[t]{3}{*}{ Idade } & $<19$ & $65(19,3)$ \\
\hline & {$[19 ; 21[$} & $139(41,4)$ \\
\hline & $\geq 21$ & $132(39,3)$ \\
\hline \multirow[t]{5}{*}{ Ano de curso } & $1^{0 *}$ & $109(32,3)$ \\
\hline & $2^{\circ *}$ & $92(27,3)$ \\
\hline & $3^{\circ *}$ & $85(25,2)$ \\
\hline & $4^{\circ}+$ & $38(11,3)$ \\
\hline & $5^{\circ}+$ & $13(3,9)$ \\
\hline \multirow[t]{5}{*}{ Residência (com quem vive) } & Pais & $117(34,6)$ \\
\hline & Outros familiares & $24(7,1)$ \\
\hline & Amigos & $81(24,0)$ \\
\hline & Colegas & $82(24,3)$ \\
\hline & Outros & $34(10,1)$ \\
\hline \multirow[t]{2}{*}{ Proveniência } & Meio rural & $141(41,8)$ \\
\hline & Meio urbano & $196(58,2)$ \\
\hline \multicolumn{3}{|l|}{ Consumos da rede social } \\
\hline \multirow[t]{2}{*}{ Ter pelo menos 1 que consome bebidas alcoólicas } & Não & $38(11,3)$ \\
\hline & Sim & $297(88,7)$ \\
\hline \multirow[t]{2}{*}{ Ter pelo menos 1 que consome tabaco } & Não & $132(39,5)$ \\
\hline & Sim & $202(60,5)$ \\
\hline \multirow[t]{2}{*}{ Ter amigos que consomem bebidas alcoólicas } & Não & $6(1,8)$ \\
\hline & Sim & $332(98,2)$ \\
\hline \multirow[t]{2}{*}{ Ter amigos que consomem tabaco } & Não & $21(6,3)$ \\
\hline & $\operatorname{Sim}$ & $315(93,8)$ \\
\hline \multicolumn{3}{|c|}{ Consumir ou alguma vez ter consumido uma das seguintes substâncias } \\
\hline \multirow[t]{2}{*}{ Tabaco } & Não & $135(40,3)$ \\
\hline & $\operatorname{Sim}$ & $200(59,7)$ \\
\hline \multirow[t]{2}{*}{ Bebidas alcoólicas } & Não & $18(5,4)$ \\
\hline & $\operatorname{Sim}$ & $317(94,3)$ \\
\hline \multirow[t]{2}{*}{ Cannabis } & Não & $231(70,2)$ \\
\hline & $\operatorname{Sim}$ & $98(29,8)$ \\
\hline \multirow[t]{2}{*}{ Cocaína } & Não & $327(98,5)$ \\
\hline & Sim & $5(1,5)$ \\
\hline \multirow[t]{2}{*}{ Estimulantes tipo anfetamina } & Não & $323(97,3)$ \\
\hline & Sim & $9(2,7)$ \\
\hline \multirow[t]{2}{*}{ Inalantes } & Não & $328(99,4)$ \\
\hline & $\operatorname{Sim}$ & $2(0,6)$ \\
\hline \multirow[t]{2}{*}{ Sedativos ou comprimidos para dormir } & Não & $315(94,9)$ \\
\hline & Sim & $17(5,1)$ \\
\hline \multirow[t]{2}{*}{ Alucinogénios } & Não & $318(95,8)$ \\
\hline & Sim & $14(4,2)$ \\
\hline \multirow[t]{2}{*}{ Opióides } & Não & $330(99,4)$ \\
\hline & $\operatorname{Sim}$ & $2(0,6)$ \\
\hline
\end{tabular}

*: licenciatura; †: mestrado e/ou mestrado integrado 
As respostas são dadas através de uma escala do tipo Likert de cinco pontos para as questões 2 a 5 (nunca; 1 ou 2 vezes; mensalmente; semanalmente; diariamente ou quase diariamente) e de três pontos para as questões 6 e 7 (não, nunca; sim, nos últimos três meses; sim, mas não nos últimos três meses). A pontuação para cada subescala (substância) é calculada pela soma das respostas obtidas nas questões correspondentes, obtendo-se assim a pontuação de consumo de risco para cada substância. ${ }^{29}$ Considera-se que quanto maior a pontuação, maior o envolvimento do estudante com determinada substância.

A versão 3.1 do ASSIST foi a selecionada para este estudo após a comparação das versões $3.0^{28}$ e 3.1. ${ }^{30}$ No que concerne ao conteúdo e estrutura das questões e formato de resposta, não foram verificadas quaisquer diferenças. No entanto, no âmbito da linguagem utilizada, a versão 3.1 era a que apresentava maior proximidade com o Português Europeu (ex. Pergunta 2: versão 3.0 "Durante os três últimos meses, com que frequência você utilizou essa(s) substância(s) que mencionou?"; verão. 3.1: "Durante os últimos três meses, quantas vezes usou as substâncias que mencionou").

Assim, não se procedeu ao processo de tradução-retroversão uma vez que foi usada a versão em português do Brasil do ASSIST (versão 3.1), disponibilizada pela OMS. No entanto, procedeu-se à adaptação cultural para português Europeu ${ }^{30}:$ i) alteração de estruturas frásicas [ex. Pergunta 5: "Nos últimos três meses, quantas vezes não fez aquilo que era normalmente se esperaria de si devido ao uso de (...)?" para "Nos últimos três meses, quantas vezes não fez aquilo que normalmente seria esperado de si (...)"]; ii) adaptação cultural [ex. "Cocaína (coke, crack, etc.)" para "Cocaína (coca, crack, etc.)”]

\section{Pré-teste e adaptação cultural do ASSIST}

Aquando do desenvolvimento do questionário, procedeu-se à aplicação de um pré-teste, seguindo as guidelines propostas pela $\mathrm{OMS}^{31}$ com o objetivo de avaliar a adaptação cultural da versão do ASSIST utilizada. O pré-teste incluiu 19 estudantes, com uma média de idades de 22,7 $\pm 3,0$ anos. Após o preenchimento do questionário, foram realizadas entrevistas cognitivas (seguindo as guidelines mencionadas anteriormente). Através dos resultados destas entrevistas, verificou-se que as questões foram compreendidas e não ocorreram equívocos, não tendo sido necessário realizar qualquer alteração linguística.

\section{Outras medidas}

$\mathrm{Na}$ recolha de dados foi utilizado um questionário incluindo questões relacionadas com aspetos sociodemográficos e comportamentos de consumo.

Para avaliar os motivos para o consumo de álcool foi utilizado o Drinking Motives Questionnaire - Revised. Esta ferramenta foi desenvolvida com vista a examinar os comportamentos de consumos etílicos e as suas consequências em quatro domínios: motivos sociais (social motives), de coping (coping), de realce/aprimoramento (enhancement) e de conformidade (conformity). ${ }^{32}$ Para o presente estudo, foi utilizada a versão traduzida e adaptada para Portugal, composta por 18 itens dos 20 originais. ${ }^{33}$ A resposta a estes itens é realizada através de uma escala tipo Likert de cinco pontos (quase nunca/nunca; algumas vezes; metade das vezes; a maior parte das vezes; quase sempre/sempre). A pontuação deste instrumento foi obtida através da soma de todas as respostas e respetiva transformação da pontuação numa escala de zero a cem (quanto maior a pontuação, mais elevado o motivo de consumo para determinado domínio).

Os quatro domínios apresentaram uma consistência interna adequada $(0,748 \leq \alpha$ de Cronbach $\leq 0,902)$ na amostra em estudo.

No âmbito dos consumos tabágicos, introduziu-se o teste de Fagerström para a dependência nicotínica (TFDN) para a avaliação desta dependência. A sua utilização é recomendada pela Circular Normativa $n^{\circ} 26 / D S P P S,{ }^{34}$ encontrando-se validado para Portugal. ${ }^{35}$ No presente estudo, esta medida apresentou uma consistência interna satisfatória $(\alpha$ de Cronbach $=0,651)$.

\section{Análise de dados}

Para avaliar a estrutura das diferentes subescalas do ASSIST, procedeu-se a uma análise fatorial exploratória (AFE) usando o método de Fatorização do Eixo Principal (MFEP). Aplicou-se o teste de esfericidade de Bartlett para testar a hipótese da matriz de correlações ser diferente da matriz identidade e determinou-se a medida de adequação da amostragem de Kaiser-Meyer-Olkin (KMO) para testar a validade da análise fatorial. Para proceder à extração dos fatores foi utilizada a rotação Varimax. Para determinar o número de fatores a extrair, usou-se o critério de Kaiser, ${ }^{36}$ a percentagem da variância explicada e o critério scree-plot (observação gráfica). ${ }^{37}$ Após a decisão do número de fatores a reter, avaliaram-se as cargas fatoriais de cada item e as respetivas comunalidades $\left(h^{2}\right)$. A estrutura fatorial foi também examinada, adicionalmente, usando o método de Análise de Componentes Principais (ACP) a fim de verificar se existiam diferenças associadas ao método aplicado. Por não terem sido obtidas estruturas fatoriais distintas usando os diferentes métodos apenas são apresentados na secção dos resultados dados referentes ao MFEP, uma vez que este método se apresenta como o mais confiável. ${ }^{38}$

Com o objetivo de se avaliar o ajuste do modelo fatorial, calcularam-se os índices seguidamente apresentados, utilizando-se como cut-offs aceitáveis para o ajustamento dos dados os valores apresentados: Goodness of Fit Index $(\mathrm{GFI}) \geq 0,90$, adjusted $\mathrm{GFI}$ (AGFI) $\geq 0,90^{39}$ e Standardized Root Mean Square Residual (SRMR) $\leq 0,08 .{ }^{40}$ Para o cálculo destes, foram utilizadas as fórmulas explanadas por Marôco ${ }^{37}$ com recurso ao software Microsoft Office Excel 2016. Recorreu-se, ainda, ao método da máxima verosimilhança para avaliar a adequação do ajuste do modelo aos dados. ${ }^{37}$

Para avaliar a confiabilidade dos modelos obtidos, foram calculados os coeficientes de correlação item-total e inter-item e para a avaliação da consistência interna foram 
calculados os valores a de Cronbach, considerando-se uma consistência interna adequada para valores de $a$ de Cronbach superiores ou iguais a $0,700 .{ }^{41}$

No que concerne à avaliação da validade convergente, foram calculados os coeficientes de correlação de Pearson (r) para determinar a associação entre as subescalas 'Tabaco', 'Bebidas Alcoólicas' e 'Cannabis' e outras variáveis relativas ao consumo de substâncias, considerando a distribuição normal dos dados. Por sua vez, a associação entre variáveis dicotómicas e as pontuações obtidas em cada uma das subescalas do ASSIST foi determinada usando o coeficiente de ponto bisserial (rpb). Para o efeito, as variáveis dicotómicas foram codificadas (e.g. 0-Não; 1-Sim e no caso do TFDN: 0-Pouco dependente; 1-Muito dependente).

Para a AFE, análise da confiabilidade e validade convergente apenas foram analisadas as subescalas do ASSIST para 'Tabaco', 'Bebidas Alcoólicas' e 'Cannabis' por não existirem dados suficientes para analisar para as restantes substâncias (proporção de consumir ou alguma vez ter consumido $\leq 5,1 \%$, ver Tabela 1 ).

Para a análise de dados utilizou-se um nível de significância (a) de 0,05. A análise de dados foi realizada através do IBM SPSS Statistics, versão 23.

\section{RESULTADOS}

A análise descritiva das pontuações observadas nas diferentes subescalas do ASSIST podem ser observadas na Tabela 2.

\section{Validade fatorial}

Os valores de $\mathrm{KMO}$ foram observados para determinar a adequação da amostra para a análise fatorial. Valores su- periores a 0,60 são requeridos e, na amostra, os valores de KMO obtidos foram de 0,825, de 0,720 e de 0,788 para 'Tabaco', 'Bebidas Alcoólicas' e 'Cannabis' respetivamente, consequentemente a análise fatorial é apropriada para os dados. ${ }^{42}$ Ainda, os testes de esfericidade de Bartlett foram significativos $\left(\chi_{(10)}^{2}=660532 ; p<0,001, \chi_{(15)}^{2}=239137\right.$; $p<0,001$ e $\chi_{(15)}^{2}=439$ 378; $p<0,001$, para 'Tabaco', 'Bebidas Alcoólicas' e 'Cannabis' respetivamente), mostrando que a AFE é executável. ${ }^{42}$ Os resultados do MFEP sugeriram a extração de um fator que explica $63,0 \%$ da variância explicada referentes a 'Tabaco', dois fatores que explicam $56,5 \%$ para 'Bebidas Alcoólicas' e um fator que explica 48,8\% para 'Cannabis' (Tabela 3).

Quanto aos pesos fatoriais obtidos nos modelos apresentados, para os itens relacionados com 'Tabaco', os pesos fatoriais variaram entre 0,368 e 0,857. O item 'Problemas de saúde, sociais, legais ou financeiros (últimos três meses)', foi o que apresentou menor peso fatorial no modelo apresentado. Para os itens referentes a 'Bebidas Alcoólicas', através da aplicação de uma rotação Varimax, os pesos fatoriais variaram entre 0,399 e 0,830. Nesta subescala, o item 'Não fazer o que seria esperado devido ao uso de substância (últimos três meses)' apresentou uma carga fatorial de 0,399. Já nos itens referentes a 'Cannabis', os pesos fatoriais variaram entre 0,456 e 0,712 (Tabela 4).

Observando o ajuste da estrutura fatorial, verificou-se que o modelo para 'Tabaco' não se ajusta aos dados $\left(\chi^{2}{ }_{(5)}\right.$ $=21,402 ; p=0,001)$, podendo ser sugestivo a remoção do item 'Problemas de saúde, sociais, legais ou financeiros (últimos três meses)' neste modelo. Por outro lado, o modelo para 'Bebidas Alcoólicas' ajusta-se aos dados $\left(\chi_{(4)}^{2}\right.$ $=2,936 ; p=0,569)$, podendo ser sugestivo a não remoção

Tabela 2 - Caracterização dos consumos de substâncias, avaliando o envolvimento com as mesmas através do ASSIST ( $\mathrm{n}=338$ )

\begin{tabular}{|c|c|c|c|}
\hline Subescala ASSIST & $\begin{array}{c}\text { Média } \\
\text { (desvio-padrão) }\end{array}$ & $\begin{array}{l}\text { Score } \\
\text { n (\%) }\end{array}$ & \\
\hline \multirow[t]{2}{*}{ Tabaco } & \multirow{2}{*}{$3,9(7,0)$} & < 4 (nenhuma intervenção) & $222(73,5)$ \\
\hline & & $\geq 4$ (necessita de intervenção) & $80(26,5)$ \\
\hline \multirow[t]{2}{*}{ Bebidas alcoólicas } & \multirow{2}{*}{$5,6(5,5)$} & < 11 (nenhuma intervenção) & $264(87,1)$ \\
\hline & & $\geq 11$ (necessita de intervenção) & $39(12,9)$ \\
\hline \multirow[t]{2}{*}{ Cannabis } & \multirow{2}{*}{$1,7(4,2)$} & < 4 (nenhuma intervenção) & $256(85,3)$ \\
\hline & & $\geq 4$ (necessita de intervenção) & $44(14,7)$ \\
\hline \multirow[t]{2}{*}{ Cocaína } & \multirow{2}{*}{$0,2(1,5)$} & < 4 (nenhuma intervenção) & $294(98,0)$ \\
\hline & & $\geq 4$ (necessita de intervenção) & $6(2,0)$ \\
\hline \multirow[t]{2}{*}{ Estimulantes tipo anfetamina } & \multirow{2}{*}{$0,2(0,5)$} & < 4 (nenhuma intervenção) & $296(98,7)$ \\
\hline & & $\geq 4$ (necessita de intervenção) & $4(1,3)$ \\
\hline \multirow[t]{2}{*}{ Inalantes } & \multirow{2}{*}{$0,1(1,4)$} & < 4 (nenhuma intervenção) & $298(99,3)$ \\
\hline & & $\geq 4$ (necessita de intervenção) & $2(0,7)$ \\
\hline \multirow[t]{2}{*}{ Sedativos ou comprimidos para dormir } & \multirow{2}{*}{$0,3(1,7)$} & < 4 (nenhuma intervenção) & $293(97,3)$ \\
\hline & & $\geq 4$ (necessita de intervenção) & $8(2,7)$ \\
\hline \multirow[t]{2}{*}{ Alucinogénios } & \multirow{2}{*}{$0,2(1,8)$} & < 4 (nenhuma intervenção) & $295(98,3)$ \\
\hline & & $\geq 4$ (necessita de intervenção) & $5(1,7)$ \\
\hline \multirow[t]{2}{*}{ Opióides } & \multirow{2}{*}{$1,1(1,6)$} & < 4 (nenhuma intervenção) & $298(99,3)$ \\
\hline & & $\geq 4$ (necessita de intervenção) & $2(0,7)$ \\
\hline
\end{tabular}


Tabela 3 - Valores próprios e percentagem de variância explicada relativos a cada uma das subescalas 'Tabaco', 'Bebidas Alcoólicas' e 'Cannabis'

\begin{tabular}{lcccccc}
\hline & \multicolumn{2}{c}{ Tabaco } & \multicolumn{2}{c}{ Bebidas Alcoólicas } & \multicolumn{2}{c}{ Cannabis } \\
Fator & Eigenvalue & $\begin{array}{c}\text { \% variância } \\
\text { cumulativa }\end{array}$ & Eigenvalue & $\begin{array}{c}\text { \% variância } \\
\text { cumulativa }\end{array}$ & $\begin{array}{c}\text { Eigenvalue variância } \\
\text { cumulativa }\end{array}$ & 48,833 \\
\hline 1 & 3,151 & 63,011 & 2,038 & 38,869 & 2,930 & 64,557 \\
2 & 0,835 & 79,720 & 1,041 & 56,485 & 0,943 & 76,257 \\
3 & 0,460 & 88,921 & 0,792 & 70,470 & 0,702 & 86,238 \\
5 & 0,342 & 95,760 & 0,627 & 81,467 & 0,599 & 9,470 \\
\hline
\end{tabular}

do item 'Não fazer o que seria esperado devido ao uso de substância (últimos três meses)'. Ainda, para 'Cannabis', verificou-se que o modelo não se ajusta aos dados $\left(\chi_{(9)}^{2}=\right.$ $59,331 ; p<0,001)$.

Apesar dos itens em questão apresentarem um factor loading inferior a 0,400 (ponto de corte fundamento por Field ${ }^{42}$ através do MFEP e avaliando a qualidade destes modelos, verifica-se que estes apresentam indicadores adequados de ajustamento. Observaram-se valores de GFI de 0,989, de 0,999 e de 0,970; valores de AGFI de 0,968, de 0,997 e de 0,930 e valores de SRMR de 0,033, de 0,020 e de 0,075 (respetivamente para as subescalas 'Tabaco', 'Bebidas Alcoólicas' e 'Cannabis'), pelo que os itens referidos anteriormente não serão removidos em análises posteriores.

Assim, as subescalas 'Tabaco' (cinco itens) e 'Cannabis' (seis itens) foram compostas por um fator, denominando-se 'Consumos e problemas relacionados com Tabaco / Cannabis'. Para a subescala 'Bebidas Alcoólicas', esta foi composta por dois fatores, sendo estes denominados 'Problemas relacionados com consumo de álcool' e 'Consumo de álcool', respetivamente para o fator 1 (quatro itens) e fator 2 (dois itens).

\section{Confiabilidade}

Na Tabela 5 podem ser observados dados referentes à avaliação da confiabilidade do ASSIST.

Relativamente à subescala 'Tabaco', os itens apresentaram uma correlação item-total entre os 0,347 e os 0,789 e um valor $a$ de Cronbach se item eliminado entre os 0,783 e os 0,883 . No geral, este fator apresenta uma consistência interna adequada ( $a$ de Cronbach $=0,842$ ). $O$ item 'Problemas de saúde, sociais, legais ou financeiros (últimos três meses)' foi o que apresentou uma menor correlação item-total e um valor de $a$ de Cronbach se item eliminado superior ao a de Cronbach do fator. Ainda, os coeficientes de correlação inter-item para 'Tabaco' variaram entre 0,279 e 0,786 .

No âmbito de 'Bebidas Alcoólicas', os fatores apresentaram um $a$ de Cronbach de 0,641 e 0,556 (fator 1 e fator 2 , respetivamente). Os valores de correlação item-total variaram entre 0,334 e 0,501 e os valores de $a$ de Cronbach se item eliminado entre os 0,603 e os 0,662. Os valores de coeficiente de correlação inter-item variaram entre 0,112 e 0,387 .

No que concerne à subescala de 'Cannabis' do ASSIST, observou-se uma consistência interna de 0,788 ( $a$ de Cronbach). A correlação item-total neste fator variou entre os

Tabela 4 - Pesos fatoriais e comunalidades $\left(\mathrm{h}^{2}\right)$ de cada item nos fatores retidos por substância, após uma AFE com extração dos fatores pelo método de Fatorização pelo Eixo Principal

\begin{tabular}{|c|c|c|c|c|c|c|c|}
\hline \multirow[b]{2}{*}{ Item } & \multirow{2}{*}{$\begin{array}{l}\text { Tabaco } \\
\text { Fator 1ा }\end{array}$} & \multirow[t]{2}{*}{$h^{2}$} & \multicolumn{2}{|c|}{ Bebidas Alcoólicas* } & \multirow[t]{2}{*}{$h^{2}$} & \multirow{2}{*}{$\begin{array}{l}\text { Cannabis } \\
\text { Fator } 1\end{array}$} & \multirow[t]{2}{*}{$h^{2}$} \\
\hline & & & Fator $1 a$ & Fator $2 \bullet$ & & & \\
\hline Utilização de substância (últimos 3 meses) & 0,857 & 0,734 & 0,136 & 0,432 & 0,205 & 0,666 & 0,444 \\
\hline $\begin{array}{l}\text { Desejo/vontade de consumir substância } \\
\text { (últimos } 3 \text { meses) }\end{array}$ & 0,857 & 0,735 & 0,173 & 0,830 & 0,719 & 0,712 & 0,506 \\
\hline $\begin{array}{l}\text { Problemas de saúde, sociais, legais ou } \\
\text { financeiros (últimos } 3 \text { meses) }\end{array}$ & 0,368 & 0,135 & 0,775 & 0,023 & 0,602 & 0,456 & 0,208 \\
\hline $\begin{array}{l}\text { Não fazer o que seria esperado devido ao } \\
\text { uso de substância (últimos } 3 \text { meses) }\end{array}$ & N/A & N/A & 0,399 & 0,178 & 0,191 & 0,625 & 0,391 \\
\hline $\begin{array}{l}\text { Expressão de preocupação devido a } \\
\text { consumo por parte de amigo/familiar/outro }\end{array}$ & 0,756 & 0,572 & 0,488 & 0,355 & 0,364 & 0,614 & 0,377 \\
\hline $\begin{array}{l}\text { Experimentar diminuir consumo de } \\
\text { substância sem sucesso }\end{array}$ & 0,783 & 0,612 & 0,444 & 0,278 & 0,275 & 0,642 & 0,412 \\
\hline
\end{tabular}

N/A: não aplicado; *rotação Varimax. ๆ: Fator 1 (consumos e problemas relacionados com tabaco); x: Fator 1 (problemas relacionados com consumo de álcool); $\bullet$ Fator 2 (consumo de álcool); : : Fator 1 (consumos e problemas relacionados com cannabis)

A negrito, encontram-se os valores de cargas fatoriais referentes à subescala 'Bebidas Alcoólicas', referindo-se a que fator o item se correlacionada melhor. 
Tabela 5 - Consistência interna para 'Tabaco', 'Bebidas Alcoólicas' e 'Cannabis'

\begin{tabular}{|c|c|c|c|c|c|c|}
\hline \multirow[b]{2}{*}{ Item } & \multicolumn{2}{|c|}{ Tabaco } & \multicolumn{2}{|c|}{ Bebidas Alcoólicas* } & \multicolumn{2}{|c|}{ Cannabis } \\
\hline & $\begin{array}{l}\text { Correlação } \\
\text { item-total }\end{array}$ & $\begin{array}{l}\text { Alpha de } \\
\text { Cronbach } \\
\text { se item } \\
\text { eliminado }\end{array}$ & $\begin{array}{l}\text { Correlação } \\
\text { item-total }\end{array}$ & $\begin{array}{l}\text { Alpha de } \\
\text { Cronbach } \\
\text { se item } \\
\text { eliminado }\end{array}$ & $\begin{array}{l}\text { Correlação } \\
\text { item-total }\end{array}$ & $\begin{array}{c}\text { Alpha de } \\
\text { Cronbach } \\
\text { se item } \\
\text { eliminado }\end{array}$ \\
\hline Utilização de substância (últimos 3 meses) & 0,787 & 0,786 & 0,334 & 0,662 & 0,598 & 0,722 \\
\hline $\begin{array}{l}\text { Desejo/vontade de consumir substância } \\
\text { (últimos } 3 \text { meses) }\end{array}$ & 0,789 & 0,783 & 0,449 & 0,627 & 0,619 & 0,716 \\
\hline $\begin{array}{l}\text { Problemas de saúde, sociais, legais ou } \\
\text { financeiros (últimos } 3 \text { meses) }\end{array}$ & 0,347 & 0,883 & 0,416 & 0,638 & 0,399 & 0,779 \\
\hline $\begin{array}{l}\text { Não fazer o que seria esperado devido ao } \\
\text { uso de substância (últimos } 3 \text { meses) }\end{array}$ & N/A & $\mathrm{N} / \mathrm{A}$ & 0,349 & 0,659 & 0,542 & 0,746 \\
\hline $\begin{array}{l}\text { Expressão de preocupação devido a } \\
\text { consumo por parte de amigo/familiar/outro }\end{array}$ & 0,704 & 0,809 & 0,501 & 0,603 & 0,548 & 0,735 \\
\hline $\begin{array}{l}\text { Experimentar diminuir consumo de } \\
\text { substância sem sucesso }\end{array}$ & 0,728 & 0,803 & 0,434 & 0,629 & 0,541 & 0,738 \\
\hline Alpha de Cronbach & --- & 0,842 & --- & 0,671 & --- & 0,788 \\
\hline
\end{tabular}

N/A: não aplicado; * rotação Varimax

0,399 e os 0,619 e o $a$ de Cronbach se item eliminado entre os 0,716 e os 0,779 . Ainda, os valores de coeficiente de correlação inter-item para esta subescala variaram entre 0,205 e 0,630 .

\section{Validade convergente}

$\mathrm{Na}$ Tabela 6 podem ser observadas as correlações entre as subescalas do ASSIST 'Tabaco', 'Bebidas Alcoólicas' e 'Cannabis' e as variáveis relativas a consumos.

De uma forma geral, verificaram-se associações estatisticamente significativas entre apresentar comportamentos de binge-drinking e as subescalas do ASSIST ('Tabaco': $\mathrm{rpb}=0,342 ; p<0,001$; 'Bebidas Alcoólicas': rpb = 0,455; $p$ $<0,001$ e 'Cannabis': rpb $=0,262$; $p<0,001$ ) e ser consumidor atual de tabaco e as subescalas do ASSIST ('Tabaco': $\mathrm{rpb}=0,766 ; p<0,001$; 'Bebidas Alcoólicas': rpb = 0,405; $p$ $<0,001$ e 'Cannabis': rpb =0,459; $p<0,001)$. Ainda, todos os motivos de consumo etílicos (avaliados pelos diferentes domínios do DMQ-R), apresentaram associações estatisticamente significativas com as subescalas 'Tabaco', 'Bebidas Alcoólicas' e 'Cannabis'.

\section{DISCUSSÃO}

O presente estudo adaptou culturalmente e analisou a estrutura fatorial, a confiabilidade e a validade convergente da versão portuguesa do ASSIST (versão 3.1), ${ }^{30}$ referente a 'Tabaco', 'Bebidas Alcoólicas' e 'Cannabis'. Esta medida apresentou adequadas características psicométricas usando uma amostra de estudantes do ensino superior.

No que concerne às subescalas 'Cocaína'; 'Estimulantes do tipo anfetaminas'; 'Inalantes'; 'Sedativos ou comprimidos para dormir'; 'Alucinogénios'; e 'Opióides' onde não foi possível o estudo das propriedades psicométricas, esta impossibilidade deveu-se à baixa proporção de consumo

Tabela 6 - Correlações entre as subescalas do ASSIST 'Tabaco', 'Bebidas Alcoólicas' e 'Cannabis' e outras medidas de avaliação do consumo de substâncias (DMQ-R e teste de Fagerström)

\begin{tabular}{|c|c|c|c|}
\hline & $\begin{array}{l}\text { ASSIST } \\
\text { Tabaco }\end{array}$ & $\begin{array}{c}\text { ASSIST } \\
\text { Bebidas Alcoólicas }\end{array}$ & $\begin{array}{l}\text { ASSIST } \\
\text { Cannabis }\end{array}$ \\
\hline Ser consumidor atual de álcool & $0,144^{*}$ & $0,276^{\text {t** }}$ & 0,104 \\
\hline Apresentar comportamentos de binge-drinking & $0,342^{* *}$ & $0,455^{\text {t* }}$ & $0,262^{* *}$ \\
\hline Ser consumidor atual de tabaco & $0,766^{\text {** }}$ & $0,405^{* *}$ & $0,459^{*+*}$ \\
\hline Dependência nicotínica & $0,303^{*}$ & 0,174 & 0,288 \\
\hline DMQ-R Enhancement & $0,330^{*+*}$ & $0,288^{* * *}$ & $0,283^{* * *}$ \\
\hline DMQ-R Coping & $0,311^{* *}$ & $0,308^{\text {**t }}$ & $0,194^{* *}$ \\
\hline DMQ-R Conformity & $0,134^{*}$ & $0,172^{* *}$ & $0,161^{\text {** }}$ \\
\hline DMQ-R Social & $0,280^{*+*}$ & $0,269^{* *}$ & $0,273^{* *}$ \\
\hline ASSIST Tabaco & 1,000 & $0,582^{* *}$ & $0,627^{*+*}$ \\
\hline ASSIST Bebidas Alcoólicas & -- & 1,000 & $0,468^{* *}$ \\
\hline ASSIST Cannabis & --- & -- & 1,000 \\
\hline
\end{tabular}

*: a correlação é significativa no nível 0,05 (bilateral); **: a correlação é significativa no nível 0,01 (bilateral) 
observada na amostra. Outros estudos verificaram esta dificuldade, quando aplicados à população geral ${ }^{23,26}$ ou em populações específicas (ex. estudantes do ensino superior). ${ }^{24}$

Nas análises da estrutura fatorial e da consistência interna, o item 'Problemas de saúde, sociais, legais ou financeiros (últimos três meses)' apresentou um baixo peso fatorial $(<0,400)$ e um $a$ de Cronbach se item eliminado superior ao a de Cronbach do fator, sendo sugestivo da exclusão deste item. Este resultado poderá ser devido aos problemas experienciados pelo consumo de tabaco (nos âmbitos financeiros, sociais, legais e de saúde), não serem entendidos pelos estudantes como consequência dos próprios consumos.

Neste contexto, no que diz respeito às consequências para a saúde, estes problemas desenvolvem-se a médio/ longo prazo e por vezes de forma assintomática, ${ }^{43}$ podendo influenciar as perceções dos estudantes, subestimando a identificação dos problemas que advém do consumo de tabaco. No âmbito social, a definição do auto-conceito de consumo (ex. fumador, fumador regular, fumador social) por parte do estudante, é influenciada pela quantidade e frequência com que fumam e pela perceção dos problemas associados. ${ }^{44,45}$ Ainda, o consumo de tabaco, sob a forma de fumar socialmente, ${ }^{45}$ é comum em ambiente académi$\mathrm{co}^{46} \mathrm{e}$, consequentemente, o ambiente social (pressão de pares), poderá fomentar a experimentação e/ou iniciação ao consumo do tabaco. ${ }^{47}$ Além disso, no que concerne aos problemas financeiros associados ao tabaco, as perceções dos estudantes poderão ser influenciadas pela possível existência de dependência financeira (progenitor - estudante), pelas condições económicas de compra dos estudantes $^{48}$ e pela frequência e quantidade de consumo. No âmbito de problemas legais, estas perceções poderão estar influenciadas com o próprio auto-conceito do estudante $^{49}$ e adesão e não adesão a políticas antitabágicas em certos estabelecimentos (ex. edifícios públicos, campus universitário, restaurantes, bares, etc.). ${ }^{49,50}$

Deste modo, considerando as relações entre os problemas de saúde, sociais, legais ou financeiros com os consumos abusivos e os resultados obtidos nos indicadores de qualidade do modelo apresentado (GFI, AGFI e SRMR), estes poderão fundamentar a não exclusão do item.

Quanto à validade fatorial para 'Tabaco' e 'Cannabis', estruturas fatoriais com um fator foram observadas noutros estudos. ${ }^{24}$ No âmbito da estrutura fatorial da 'Bebidas Alcoólicas', obtiveram-se dois fatores. Porém, num estudo de validação obteve-se um fator que explicou $35 \%$ da variância e com uma consistência interna de $0,760 .{ }^{23}$

$\mathrm{Na}$ amostra em estudo, a subescala do 'Bebidas Alcoólicas' dividiu-se em 2 fatores: 'Problemas relacionados com o consumo de álcool' e 'Consumo de álcool. Os fatores obtidos poderão ser explicados e corroborados pela relação entre o consumo de álcool com os problemas decorrentes do seu consumo. Neste sentido, existe uma associação entre os problemas de consumo com o beber para regular efeitos negativos (motivos de coping) e com o consumir para aprimorar positivamente o estado de humor/disposição (motivos de aprimoramento). ${ }^{51}$ Ainda, verifica-se que os indivíduos que experienciam consequências negativas estão dispostos a experienciá-las novamente, sendo sugestivo que os indivíduos consideram as consequências negativas como um meio para atingir consequências positivas. $^{52}$

Neste enquadramento, a relação entre o consumo de álcool com os problemas relacionados, é mediada quer pelo tipo de bebidas consumidas (onde se observam consequências pessoais e sociais negativas), quer pela idade (em que o consumo precoce associa-se a consequências negativas pessoais e sociais). ${ }^{53}$

No âmbito da confiabilidade, foram observados noutros estudos consistências internas, avaliadas pelo $a$ de Cronbach, entre os $0,740^{54}$ e os $0,860^{23}$ para 'Tabaco'; e entre os $0,730^{24}$ e os $0,870^{23}$ para 'Cannabis'. Quanto à consistência interna na subescala 'Bebidas Alcoólicas', outros estudos verificaram uma consistência interna entre os $0,660^{54}$ e os $0,840^{55}$. Na presente amostra, semelhantes características de consistência interna foram observadas.

$\mathrm{Na}$ validade convergente das subescalas estudadas, verificou-se que os estudantes com maiores níveis de comportamentos de consumo de risco de tabaco, são os que: consomem álcool atualmente; apresentam comportamentos de binge-drinking; apresentam maior dependência nicotínica; e apresentam maiores níveis de motivos de consumos etílicos. Quanto às bebidas alcoólicas, os estudantes que apresentam maiores níveis de consumos de riscos são os que: apresentam comportamentos binge-drinking; consomem atualmente tabaco; e apresentam maiores níveis de motivos de consumos etílicos. Já no consumo de cannabis, os estudantes com maiores níveis de comportamentos de consumo de risco, são os que: apresentam comportamentos binge-drinking; consomem atualmente tabaco; e apresentam maiores motivos de consumos etílicos. Estas associações estatisticamente significativas, entre o consumo de diferentes substâncias, poderão ser explicadas pelos resultados verificados por Bravo et al, ${ }^{56}$ onde se observaram associações significativas entre as crenças relativas ao consumo de álcool, como parte da experiência académica, com os diferentes motivos de consumos etílicos (avaliado pelo DMQ-R) e com os consumos do tipo binge-drinking.

Ainda na validade convergente, verificaram-se associações positivas significativas entre as diferentes subescalas do ASSIST. Estas associações poderão ser indicadoras da presença de policonsumos. De acordo com a tipologia de policonsumos definida pela EMCDDA,${ }^{57}$ verificaram-se, nesta amostra, policonsumos do tipo A (álcool e tabaco) e do tipo B (cannabis concomitantemente com álcool e/ou tabaco), indo ao encontro com o observado em diferentes estudos. ${ }^{58,59}$

\section{Limitações do estudo}

O presente estudo apresenta como principais limitações a sua restrição geográfica, que poderá influenciar a 
avaliação de consumos de risco. Ainda, neste estudo, apenas foi possível proceder à avaliação da consistência interna e validade convergente, não tendo sido possível a avaliação da validade divergente, para uma avaliação mais abrangente da validade de construto.

Ademais, a baixa proporção de consumos no que concerne a outras substâncias, fez com que não tornasse viável o estudo das propriedades psicométricas para as restantes subescalas. Esta baixa proporção de consumos, poderá ser devido ao viés da resposta socialmente desejável, por se questionar assuntos sensíveis como consumo de substâncias. ${ }^{60}$

Desta forma, alargar o estudo a outras instituições de ensino superior, bem como o estudo das propriedades psicométricas da escala em outras populações, mais especificamente entre amostras clínicas com perturbações aditivas, poderão representar medidas para minimizar as limitações referidas anteriormente.

\section{CONCLUSÃO}

Com este estudo foi possível adaptar culturalmente o questionário ASSIST versão 3.1 para português Europeu, verificando-se adequadas características fatoriais, consistência interna e validade convergente para as subescalas 'Tabaco', 'Bebidas Alcoólicas' e 'Cannabis'. No entanto, não foi possível avaliar as propriedades psicométricas das subescalas 'Cocaína'; 'Estimulantes do tipo Anfetaminas'; 'Inalantes'; 'Sedativos ou comprimidos para dormir'; 'Alucinogénios'; e 'Opióides', devido à baixa proporção de con-

\section{REFERÊNCIAS}

1. O'Malley PM, Johnston LD. Epidemiology of alcohol and other drug use among American college students. J Stud Alcohol. 2002:23-39.

2. Nolen-Hoeksema S. Gender differences in risk factors and consequences for alcohol use and problems. Clin Psychol Rev. 2004;24:981-1010.

3. World Health Organization. Toolkit for oral health professionals to deliver brief tobacco interventions in primary care [Internet]. 2017. [consultado 2018 mar 3]. Disponível em: http://apps.who.int/iris/bitstre am/10665/255628/1/9789241512510-eng.pdf.

4. World Health Organization. WHO Expert Committee on Problems Related to Alcohol Consumption [Internet]. 2007. [consultado 2018 mar 3]. Disponivel em: http://www.who.int/substance_abuse/expert_ committee alcohol trs944.pdf.

5. Kilmer JR, Hunt SB, Lee CM, Neighbors C. Marijuana use, risk perception, and consequences: Is perceived risk congruent with reality? Addict Behav. 2007;32:3026-33.

6. Baer JS, Kivlahan DR, Marlatt GA. High-risk drinking across the transition from high school to college. Alcohol Clin Exp Res. 1995;19:54-61.

7. Rimal RN, Real K. How behaviors are influenced by perceived norms. Communic Res. 2005;32:389-414.

8. Lamont AE, Woodlief D, Malone PS. Predicting high-risk versus higherrisk substance use during late adolescence from early adolescent risk factors using Latent Class Analysis. Addict Res Theory. 2014;22:78-89.

9. Borsari B, Carey KB. Peer influences on college drinking: a review of the research. J Subst Abuse. 2001;13:391-424.

10. Reed MB, McCabe C, Lange JE, Clapp JD, Shillington AM. The relationship between alcohol consumption and past-year smoking initiation in a sample of undergraduates. Am J Drug Alcohol Abuse. 2010;36:202-7.

11. Instituto Nacional de Estatística. Inquérito Nacional de Saúde 2014 [Internet]. 2016. [consultado 2018 jun 2]. Disponível em: https://www.ine.pt/xportal/xmain?xpid=INE\&xpgid=ine_ publicacoes\&PUBLICACOESpub_boui=263714091\&PUBLICACOESm odo $=2$.

12. Observatório Europeu da Droga e da Toxicodependência -EMCDDA. sumo observada na amostra. Pôde ainda verificar-se que a utilização das subescalas de 'Tabaco', 'Bebidas Alcoólicas' e 'Cannabis', poderá ser adequada para a avaliação do envolvimento dos estudantes do ensino superior com essas substâncias. Futuros trabalhos deverão explorar o poder discriminativo, entre outras propriedades para detetar consumos de risco nesta população. Além disso, deverão ainda incidir noutras metodologias, aprofundando o estudo da validade de construto.

\section{PROTECÇÃO DE PESSOAS E ANIMAIS}

Os autores declaram que os procedimentos seguidos estavam de acordo com os regulamentos estabelecidos pelos responsáveis da Comissão de Investigação Clínica e Ética e de acordo com a Declaração de Helsínquia da Associacção Médica Mundial.

\section{CONFIDENCIALIDADE DOS DADOS}

Os autores declaram ter seguido os protocolos do seu centro de trabalho acerca da publicação de dados.

\section{CONFLITO DE INTERESSES}

Os autores declaram não ter nenhum conflito de interesses na realização do presente artigo.

\section{FONTES DE FINANCIAMENTO}

Os autores declaram não ter recebido subsídios ou bolsas para a elaboração do artigo.

Relatório Europeu sobre Drogas 2017: Tendências e evoluções [Internet]. Serviço das Publicações da União Europeia. 2017. [consultado 2018 jun 2]. Disponível em: http://www.emcdda.europa.eu/ system/files/publications/4541/TDAT17001PTN.pdf.

13. World Health Organization. Ottawa Charter for Health Promotion [Internet]. 1986. [consultado 2018 fev 1]. Disponível em: http://www. euro.who.int/_data/assets/pdf_file/0004/129532/Ottawa_Charter.pdf.

14. World Health Organization. Declaration of Alma-Ata: International Conference on Primary Health Care [Internet]. 1978. [consultado 2018 Jun 3]. Disponível em: http://www.who.int/publications/almaata_ declaration_en.pdf.

15. Kraemer KM, McLeish AC, O'Bryan EM. The role of intolerance of uncertainty in terms of alcohol use motives among college students. Addict Behav. 2015;42:162-6.

16. Davoren MP, Shiely F, Byrne M, Perry IJ. Hazardous alcohol consumption among university students in Ireland: a cross-sectional study. BMJ Open. 2015;5:e006045.

17. Babor TF, Higgins-Biddle JC, Saunders JB, Monteiro MG. AUDIT The Alcohol Use Disorders Identification Test - Guidelines for Use in Primary Care [Internet]. $2^{\text {nd }}$ ed. World Health Organization; 2001. [consultado 2018 jan 16]. Disponivel em: http://www.who.int/substance_abuse/ publications/audit/en/.

18. WHO ASSIST Working Group. The Alcohol, Smoking and Substance Involvement Screening Test (ASSIST): development, reliability and feasibility. Addiction. 2002;97:1183-94.

19. Teeters JB, Borsari B, Martens MP, Murphy JG. Brief motivational interventions are associated with reductions in alcohol-impaired driving among college drinkers. J Stud Alcohol Drugs. 2015;76:700-9.

20. Yurasek AM, Borsari B, Magill M, Mastroleo NR, Hustad JT, Tevyaw TO, et al. Descriptive norms and expectancies as mediators of a brief motivational intervention for mandated college students receiving stepped care for alcohol use. Psychol Addict Behav. 2015;29:1003-11.

21. Denering LL, Spear SE. Routine use of screening and brief intervention for college students in a university counseling center. J Psychoactive 
Drugs. 2012;44:318-24.

22. Andrade AG, Duarte PC, Barroso LP, Nishimura R, Alberghini DG, Oliveira LG. Use of alcohol and other drugs among Brazilian college students: effects of gender and age. Rev Bras Psiquiatr. 2012;34:294305.

23. Rubio Valladolid G, Martínez-Raga J, Martínez-Gras I, Ponce Alfaro G, de la Cruz Bértolo J, Jurado Barba R, et al. Validation of the Spanish version of the Alcohol, Smoking and Substance Involvement Screening Test (ASSIST). Psicothema. 2014;26:180-5.

24. Sainz MT, Rosete-Mohedano MG, Natera Rey G, Martínez Vélez NA, Carreño García S, Pérez Cisneros D, et al. Validity and reliability of the Alcohol, Smoking, and Substance Involvement Screening Test (ASSIST) in University students. Adicciones. 2016;28:19.

25. van der Westhuizen C, Wyatt G, Williams JK, Stein DJ, Sorsdahl K. Validation of the Alcohol, Smoking and Substance Involvement Screening Test in a low- and middle-income country cross-sectional emergency centre study. Drug Alcohol Rev. 2016;35:702-9.

26. Khan R, Chatton A, Nallet A, Broers B, Thorens G, Achab-Arigo S, et al. Validation of the French version of the Alcohol, Smoking and Substance Involvement Screening Test (ASSIST). Eur Addict Res. 2011;17:190-7.

27. Tavares J, Pereira AS, Gomes AA, Monteiro SM, Gomes A. Manual de psicologia do desenvolvimento e aprendizagem. Porto: Porto Editora; 2007.

28. Direcção-Geral da Saúde. Norma n 36/2012: Diagnóstico de policonsumos e intervenção breve em adolescentes e jovens [Internet]. 2014 [consultado 2016 mai 28]. Disponível em: https://www.dgs.pt/... dgs/normas...normativas/norma-n-0362012-de-30122012-png.a.

29. World Health Organization. The Alcohol, Smoking and Substance Involvement Screening Test (ASSIST) -Manual for use in primary care [Internet]. Humeniuk R, Henry-Edwards S, Ali R, Poznyak V, Monteiro MG, editors. 2003. [consultado 2017 mar 2]. Disponível em: http://www. who.int/substance_abuse/activities/en/Draft_The_ASSIST_Guidelines. pdf.

30. World Health Organization. Teste de triagem (ASSIST v. 3.1) do envolvimento com bebidas alcoólicas, fumo e fármacos [Internet]. 2010. [consultado 2018 abr 12]. Disponível em: http://www.who.int/ substance_abuse/activities/assist_3.1_portuguese.pdf.

31. World Health Organization. Process of translation and adaptation of instruments [Internet]. 2017. [consultado 2016 set 15]. Disponível em: http://www.who.int/substance_abuse/research_tools/translation/en/.

32. Cooper ML. Motivations for alcohol use among adolescents: development and validation of a four-factor model. Psychol Assess. 1994;6:117-28.

33. Fernandes-Jesus M, Beccaria F, Demant J, Fleig L, Menezes I, Scholz $U$, et al. Validation of the Drinking Motives Questionnaire - Revised in six European countries. Addict Behav. 2016;62:91-8.

34. Nunes E, Candeias A, Mendes B, Pardal C, Fonseca J, Oliveira L, et al. Programa-tipo de actuação em cessação tabágica - Circular Normativa No: 26/DSPPS [Internet]. Direcção-Geral de Saúde. 2007. [consultado 2016 dez 2]. Disponível em: http://www.dgs.pt/upload/membro.id/ ficheiros/i009309.pdf.

35. Ferreira PL, Quintal C, Lopes I, Taveira N. Teste de dependência à nicotina: validação linguística e psicométrica do teste de Fagerström. Dependência tabágica [Internet]. 2009. [consultado 2016 abr 28]. Disponível em: http://www.uc.pt/feuc/pedrof/docs/Publicacoes/ AN200908.pdf.

36. Kaiser HF. The application of electronic computers to factor analysis. Educ Psychol Meas. 1960;20:141-51.

37. Marôco J. Análise estatística com utilização do SPSS. $3^{a}$ ed. Lisboa: Edições Sílabo; 2007.

38. Njeri Ngure J. Principal component and principal axis factoring of factors associated with high population in urban areas: a case study of Juja and Thika, Kenya. Am J Theor Appl Stat. 2015;4:258.

39. Sivo SA, Fan X, Witta EL, Willse JT. The search for "Optimal" dutoff properties: fit index criteria in structural equation modeling. J Exp Educ. 2006;74:267-88.

40. Schreiber JB, Nora A, Stage FK, Barlow EA, King J. Reporting structural equation modeling and confirmatory factor analysis results: a review. J Educ Res. 2006;99:323-38.
41. Terwee CB, Bot SD, de Boer MR, van der Windt DA, Knol DL, Dekker $\mathrm{J}$, et al. Quality criteria were proposed for measurement properties of health status questionnaires. J Clin Epidemiol. 2007;60:34-42.

42. Field A. Discovering statistics using SPSS. $3^{\text {rd }}$ edition. London: Sage Publications; 2009.

43. U.S. Department of Health and Human Services. The Health Consequences of Smoking-50 Years of Progress: A Report of the Surgeon GeneraL [Internet]. Department of Health and Human Services, Centers for Disease Control and Prevention, National Center for Chronic Disease Prevention and Health Promotion, Office on Smoking and Health. 2014. [consultado 2018 fev 24]. Disponível em: https://www.surgeongeneral.gov/library/reports/50-years-of-progress/ exec-summary.pdf.

44. Lindgren KP, Neighbors C, Gasser ML, Ramirez JJ, Cvencek D. A review of implicit and explicit substance self-concept as a predictor of alcohol and tobacco use and misuse. Am J Drug Alcohol Abuse. 2017;43:23746.

45. Waters K, Harris K, Hall S, Nazir N, Waigandt A. Characteristics of socia smoking among college students. J Am Coll Heal. 2006;55:133-9.

46. Rigotti NA, Moran SE, Wechsler H. US college students' exposure to tobacco promotions: prevalence and association with tobacco use. Am J Public Health. 2005;95:138-44.

47. Eisenberg D, Golberstein E, Whitlock JL. Peer effects on risky behaviors: new evidence from college roommate assignments. J Health Econ. 2014;33:126-38.

48. Bono RS, Barnes AJ, Dick DM, Kendler KS. Drinking, cigarette smoking, and employment among American college freshmen at a four-year university. Subst Use Misuse. 2017;52:182-93.

49. Berg CJ, Lessard L, Parelkar PP, Thrasher J, Kegler MC, Escoffery C, et al. College student reactions to smoking bans in public, on campus and at home. Health Educ Res. 2011;26:106-18.

50. Baillie L, Callaghan D, Smith M, Bottorff J, Bassett-Smith J, Budgen $\mathrm{C}$, et al. A review of undergraduate university tobacco control policy process in Canada. Health Educ Res. 2009;24:922-9.

51. Merrill JE, Wardell JD, Read JP. Drinking motives in the prospective prediction of unique alcohol-related consequences in college students. J Stud Alcohol Drugs. 2014;75:93-102.

52. Wicki M, Mallett KA, Delgrande Jordan M, Reavy R, Turrisi R, Archimi $A$, et al. Adolescents who experienced negative alcohol-related consequences are willing to experience these consequences again in the future. Exp Clin Psychopharmacol. 2018;26:132-7.

53. De Bruyn S, Wouters E, Ponnet K, Van Damme J, Maes L, Van Hal G. Problem drinking among Flemish students: beverage type, early drinking onset and negative personal; \& social consequences. BMC Public Health. 2018;18:234.

54. Khan R, Chatton A, Thorens G, Achab S, Nallet A, Broers B, et al. Validation of the French version of the alcohol, smoking and substance involvement screening test (ASSIST) in the elderly. Subst Abuse Treat Prev Policy. 2012;7:14.

55. Humeniuk R, Ali R, Babor TF, Farrell M, Formigoni ML, Jittiwutikarn J, et al. Validation of the alcohol, smoking and substance involvement screening test (ASSIST). Addiction. 2008;103:1039-47.

56. Bravo AJ, Pearson MR, Pilatti A, Read JP, Mezquita L, Ibáñez MI, et al. Cross-cultural examination of college drinking culture in Spain, Argentina, and USA: measurement invariance testing of the College Life Alcohol Salience Scale. Drug Alcohol Depend. 2017;180:349-55.

57. European Monitoring Centre for Drugs and Drug Addiction. Polydrug use: patterns and responses [Internet]. Spain: European Monitoring Centre for Drugs and Drug Addiction (EMCDDA); 2009. [consultado 2018 abr 12]. Disponível em: http://www.emcdda.europa.eu/publications/ selected-issues/polydrug-use-patterns-and-responses_en.

58. Hernández-Serrano O, Font-Mayolas S, Gras ME. Polydrug use and its relationship with the familiar and social context amongst young college students. Adicciones. 2015;27:205-13.

59. Lee CK, Corte C, Stein KF. Relationships between early alcohol experiences, drinker self-schema, drinking and smoking in college students. Subst Abus. 2018;1-26.

60. Grimm P. Social desirability bias [Internet]. Wiley International Encyclopedia of Marketing. Chichester: John Wiley \& Sons, Ltd; 2010. 